\title{
A Novel Objective Approach Towards Physicians' Knowledge and Practice Patterns to Optimize Central Venous Catheter Placement: A Cross-sectional Study
}

\author{
Rakan M. AlQahtani ${ }^{1, *}$
}

${ }^{1}$ Assistant Professor, Department of Critical Care Medicine, College of Medicine, King Saud University, Riyadh, Kingdom of Saudi Arabia

\section{*Correspondence \\ dr_rknq@hotmail.com; \\ arakan@ksu.edu.sa}

(Rakan M. AlQahtani)

\begin{abstract}
Study objective: Central venous catheterization is an essential component of intensive care of critically ill patients, and proper positioning of the catheter is essential to prevent position-related complications. This study was conducted by using digital tape measurement to objectively assess clinician preferences for central venous catheter positioning based on specific position levels and landmarks on post-procedural chest radiographs. Design: A cross-sectional study using electronic questionnaire survey. Setting: Single academic teaching hospital participated in this study. Participants: The study enrolled 276 physicians from multiple clinical disciplines. Interventions: None. Measurements: A seven-level reference system labeled on a sample chest radiograph was used to identify the acceptable lower and upper limits and landmarks used to determine the optimal central venous catheter tip position as well as the pattern of clinical practices based on the specialty and level of experience of participants. Main results: Among the 276 respondents, the ratio of cumulative acceptance for the lower and upper catheter tip limit was $62 \%$ and $66.3 \%$ within a $4-\mathrm{cm}$ range below or above the carina, respectively. Intensive care unit (ICU) physicians showed a greater tendency to choose a catheter tip $4 \mathrm{~cm}$ below and $6 \mathrm{~cm}$ above the carina $(\mathrm{p}=0.004$ and 0.002 , respectively) as did experienced physicians ( $\mathrm{p}=0.007$ and $<0.001$, respectively). The commonest reason for catheter tip withdrawal was arrhythmia ( $50 \%$ of cases). Physicians in the ICU and experienced physicians were more concerned about the risk of cardiac perforation than other respondents $(\mathrm{p}<0.001$ and $<0.001$, respectively). The carina was the most commonly used landmark in $71.7 \%$ of all physicians, although $50 \%$ of radiologists also used other landmarks. Conclusions: The acceptable limit of the catheter tip is $4 \mathrm{~cm}$ above and below the carina ( -4 to +4$)$, as determined on chest radiography, without a need for tip adjustment.
\end{abstract}

\section{Keywords}

Central venous catheters, Catheter tip position, Carina, Superior vena cava, Chest X-ray, Cardiac tamponade

\section{Introduction}

The central venous catheter (CVC) is an essential component in the care of severely ill patients with various diseases and across diverse treatment environments. The $\mathrm{CVC}$ is routinely and frequently used for the administration of intravenous fluids, medications, and nutrition, as well as for advanced monitoring of hemodynamics. Confirmation of the catheter tip position in the fields of critical care medicine and anesthesia recommended by the American Society of Anesthesiologists task force practice guidelines is crucial after $\mathrm{CVC}$ insertion to prevent complications such as mechanical endocardial injury, arrhythmias, and, most seriously, cardiac tamponade, which is associated with a high mortality rate $[1,2]$. The incidence of inaccurate CVC positioning, with the catheter tip below the junction of the superior vena cava (SVC) and the right atrium (RA), has been reported to be as high as $3.3 \%$ [3].

Other practice guidelines have been issued with regard to the placement and positioning of dialysis catheters, Port-A-Caths, and peripherally inserted central venous catheters (PICCs) [47]. The National Kidney Foundation has proposed practice guidelines for the use of CVCs, and recommends that nontunneled dialysis or trialysis catheters be placed in the distal half of the SVC or at the junction of the SVC and RA, whereas the tip for long-term tunneled catheters should be placed at least at the SVC-RA junction, or even within the RA itself, to achieve maximum flow in dialysis-dependent patients [4]. Specific guidelines for the insertion and management of central venous access devices in adults, established by 
TA B L E 1. Acceptable lower CVC tip position based on the specialty and the level of experience (individual CVC tip-carina distance).

\begin{tabular}{lccccccccc} 
LA-CVC & ICU n (\%) & RAD n (\%) & IM n (\%) & Others n (\%) & p-value & Resident n (\%) & AP n (\%) & p-value & Total n (\%) \\
$\mathbf{0}$ & $6(7.7)$ & $3(4.6)$ & $24(22.2)$ & 0 & $<0.001^{*}$ & $27(14.8)$ & $6(6.45)$ & $0.04^{*}$ & $33(12)$ \\
$\mathbf{+ 2}$ & $12(15.4)$ & $15(22.7)$ & $33(30.6)$ & $12(50)$ & $0.004^{*}$ & $51(27.9)$ & $21(22.6)$ & 0.344 & $72(26)$ \\
$\mathbf{+ 4}$ & $48(61.5)$ & $39(59.1)$ & $42(38.9)$ & $9(37.5)$ & $0.004^{*}$ & $81(44.3)$ & $57(61.3)$ & $0.007^{*}$ & $130(47.1)$ \\
$+\mathbf{+ 6}$ & $12(15.4)$ & $9(13.6)$ & $9(8.3)$ & $3(12.5)$ & 0.5 & $24(13.1)$ & $9(9.7)$ & 0.405 & $33(12)$ \\
\hline Total & 78 & 66 & 108 & 24 & - & 183 & 93 & - & 276 \\
\hline
\end{tabular}

$L A$ - CVC, lower acceptable central venous catheter tip limit; RAD, radiology; IM, internal medicine; AP, attending physicians; ICU, intensive care unit. ${ }^{*} p \leq 0.05$ indicates significance.

TA B L E 2. Acceptable lower CVC tip position based on the specialty and the level of experience (cumulative CVC tip-carina distance).

\begin{tabular}{|lcccccccccc|}
\hline CLA - CVC & ICU n (\%) & RAD n (\%) & IM n (\%) & Others n (\%) & p- value & Resident n (\%) & AP n (\%) & p-value & Total \\
\hline $\mathbf{0}$ & $78(100)$ & $66(100)$ & $108(100)$ & $24(100)$ & N/A & $183(100)$ & $93(100)$ & N/A & $276(100)$ \\
$\leq+\mathbf{2}$ & $72(92.3)$ & $63(95.4)$ & $84(77.8)$ & $24(100)$ & $<0.001^{*}$ & $156(85.2)$ & $87(93.5)$ & $0.04^{*}$ & $243(88)$ \\
$\leq+4$ & $60(76.9)$ & $48(72.7)$ & $51(47.2)$ & $12(50.0)$ & $<0.001^{*}$ & $105(57.4)$ & $66(71)$ & $0.028^{*}$ & $171(62)$ \\
$\leq+\mathbf{6}$ & $12(15.4)$ & $9(13.6)$ & $9(8.3)$ & $3(12.5)$ & 0.493 & $24(13.1)$ & $9(9.7)$ & 0.405 & $33(12)$ \\
\hline
\end{tabular}

CLA - CVC, cumulative lower acceptable central venous catheter tip limit; RAD, radiology; IM, Internal medicine; AP, attending physicians; ICU, intensive care unit. ${ }^{*} p \leq 0.05$ indicates significance.

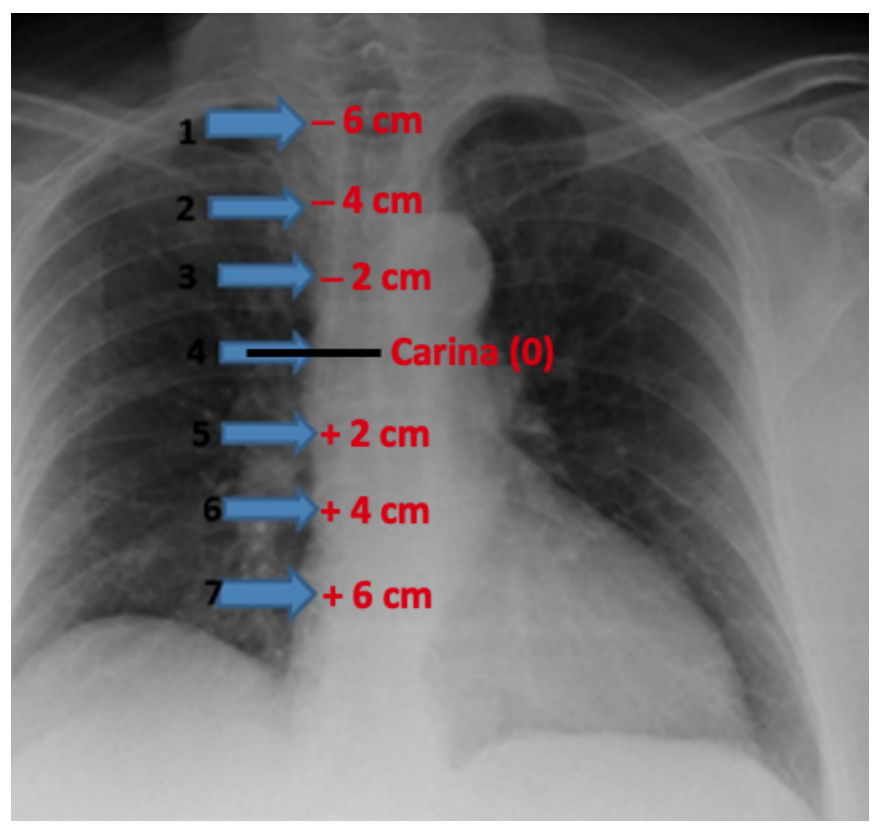

FIGURE 1. Chest radiograph with multiple levels to facilitate selection of the optimal upper and lower acceptable levels based on the distance from the carina.

a group of hematologists, indicate that the optimal position for the catheter tip is in the distal SVC or upper RA, and the carina is an approximate landmark for this position [5]. Other guidelines published for nutritionists and nurses further support the specifications for optimal CVC tip positioning $[6,7]$.

Some guidelines on catheter tip positioning recommend a post-procedural chest radiography to confirm appropriate catheter placement such as the practice guidelines for CVA
2020 by ASA Task Force [1, 5-7]. On the contrary, other guidelines such as the National Kidney Foundation suggest using CXR after the CVC insertion, but not mandatory [4].

This study was conducted with a primary objective to establish a feasible and objective approach for conventional CVC tip positioning in intensive care unit (ICU) patients, based on the CXR interpretation. This objective approach used a digital tape measurement in centimeters from the imaging software system to determine the acceptable lower and upper CVC tip position from the carina. The secondary objective was to identify and address the critical clinical aspects and controversial issues associated with catheter tip positioning in ICU.

\section{Materials and Methods}

\subsection{Study design}

This cross-sectional study used a custom-designed questionnaire survey through a Web-based application to obtain data on clinical practice patterns and knowledge of CVC positioning among physicians from an academic teaching hospital on April and May of 2020. Practice patterns referred to the acceptable upper and lower limits for the CVC tip position, as measured on chest radiography, among the physicians. In addition, we compared practice patterns based on the level of training and different medical subdisciplines for the positioning of the CVC across diverse practice settings. Additionally, the data for analysis included the most familiar landmarks or other less important ones used to determine an acceptable position of the CVC tip, the common and severe complications of unacceptable tip positioning, and the importance of tip position in the clinical setting. 


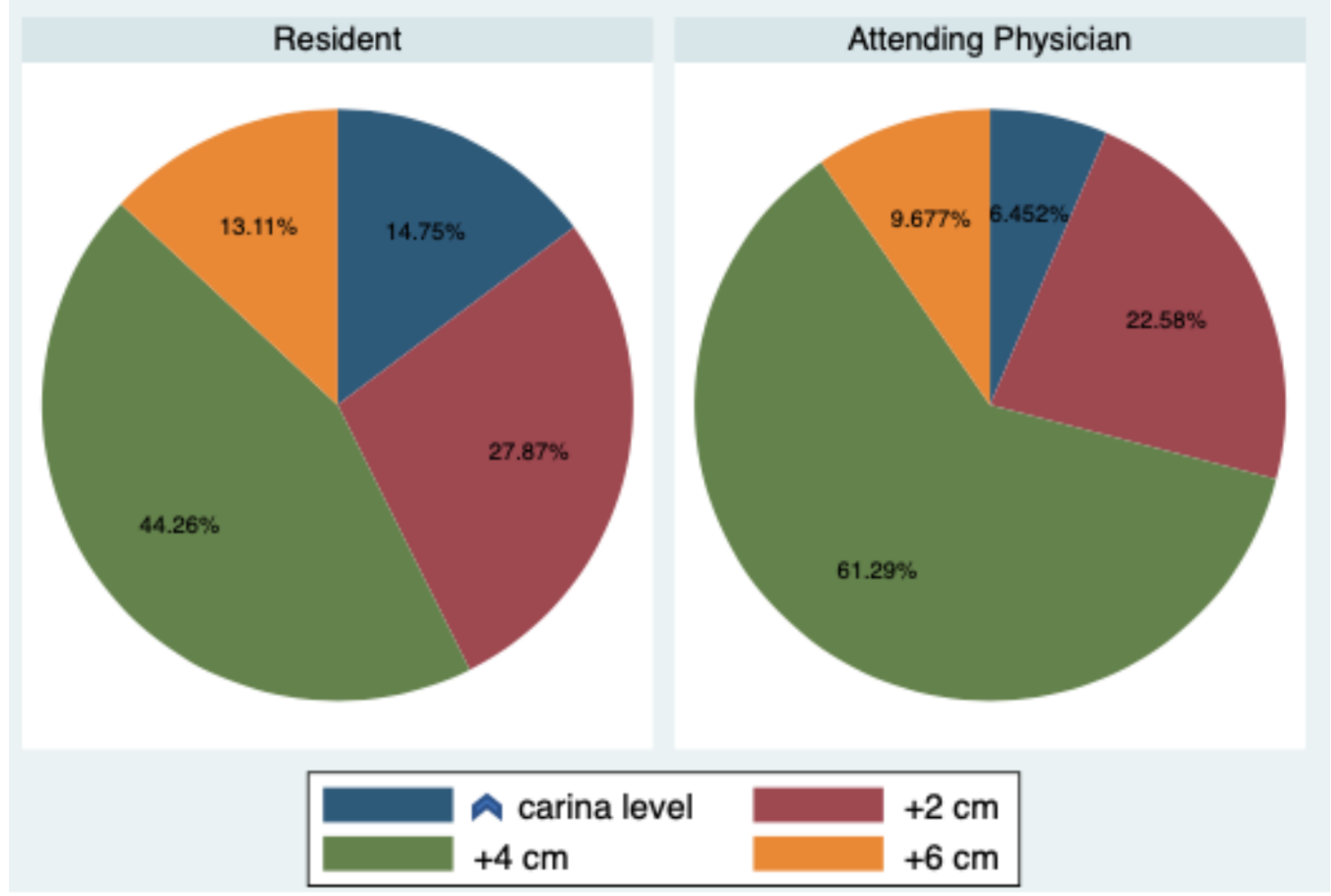

F I G U R E 2. Pie chart depicting the choice of the lower limit of central venous catheter tip position among residents and attending physicians. The attending physicians tended to choose a position $4 \mathrm{~cm}$ below the carina more often than did the residents $(\mathbf{p}=\mathbf{0 . 0 0 7})$.

\subsection{Study setting and participants}

After the initial design of the questionnaire, we conducted an intranet-based survey among physicians in two academic teaching hospitals that were affiliated to King Saud University, Riyadh, Saudi Arabia. Participants were prospectively enrolled on the basis of their medical discipline and level of experience to achieve a representative sample from different practice fields (ICU, radiology, nephrology, anesthesia, and internal medicine), and included attending physicians as well as senior trainees with at least three years of residency training who were competent and can insert conventional CVC without supervision and had completed a minimum rotation of 3 months in the ICU.

This study was approved (approval no. E-20-4766) by the institutional review board (IRB) of the health sciences college research on human subjects at King Saud University, Riyadh, Saudi Arabia.

\subsection{Questionnaire design}

A seven-level reference system, labeled on chest radiographs, was used to enable physicians to mark their upper and lower acceptable CVC tip positions. To facilitate accurate measurements in a standardized chest radiograph, we chose the carina as the reference landmark on the radiographs, with the carina defined as the " 0 " level, with three levels above and below the carina that were situated $2 \mathrm{~cm}$ apart (i.e., at 2, 4, and $6 \mathrm{~cm}$ from the carina; Fig. 1). The distances above the carina were defined as negative values, and those below the carina as positive, as follows: $-6,-4,-2,0,+2,+4$, and +6 . The following three variables were identified to determine the patterns of clinical practice and knowledge with regard to CVC tip positioning: 1) the lower limit of the CVC tip position at which the physician would consider pulling up the catheter, with additional questions on the justification for this action and its clinical importance; 2) the upper limit of the CVC tip position, with further questions on the course of action if the catheter was above the acceptable upper limit; and 3) landmarks, other than the carina, that were used to determine the optimal and acceptable CVC tip position (Table 1).

\subsection{Survey reliability and validity}

To ascertain the face validity of the key assessment tool, the questionnaire was sent to five experienced intensivists and one ICU trainee, on three separate occasions, and the survey questions were adjusted on the basis of their feedback. However, responses from the face-validity surveys were not included in the final analysis dataset. The overall test-retest reliability coefficient for the survey questionnaire was acceptable, at 0.79 . The finalized questionnaire was uploaded to 


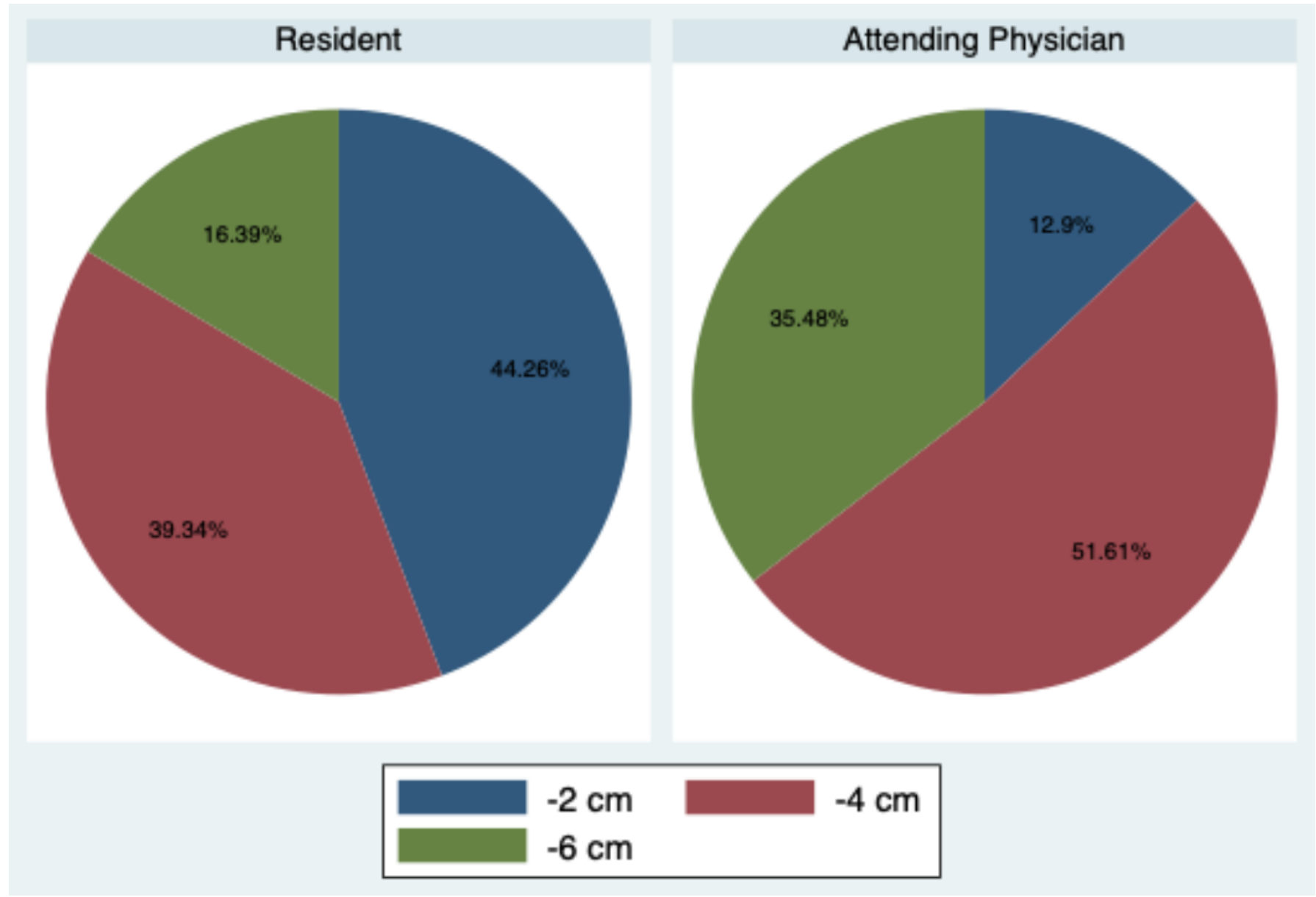

F I G U R E 3. Pie chart presenting the preference of the upper limit of central venous catheter tip position among residents and attending physicians. The attending physicians tended to choose a position $6 \mathrm{~cm}$ above the carina more often than did the residents $(\mathbf{p}<\mathbf{0 . 0 0 1})$.

TA B L E 3. Anticipated reason for catheter withdrawal below the acceptable central venous catheter tip range.

\begin{tabular}{|c|c|c|c|c|c|c|c|c|c|}
\hline & ICU n (\%) & RAD n (\%) & IM n (\%) & Others n (\%) & $p$ - value & Resident n (\%) & AP n (\%) & $p$ - value & Total n (\%) \\
\hline RA location & $21(26.9)$ & $15(22.7)$ & $27(25.0)$ & $9(37.5)$ & 0.551 & $45(24.6)$ & $27(29.3)$ & 0.42 & $72(26.1)$ \\
\hline Arrhythmias & $24(30.8)$ & $39(59.1)$ & $69(63.9)$ & $6(25.0)$ & $<0.001$ & $108(59.0)$ & $30(32.3)$ & $<0.001$ & $138(50.0)$ \\
\hline Cardiac perforation & $24(30.8)$ & $6(9.1)$ & $9(8.3)$ & $6(25.0)$ & $<0.001$ & $15(8.2)$ & $30(32.3)$ & $<0.001$ & $45(16.3)$ \\
\hline Thrombosis & $9(11.5)$ & $6(9.1)$ & $3(2.8)$ & $3(12.5)$ & 0.097 & $15(8.2)$ & $6(6.5)$ & 0.605 & $21(7.6)$ \\
\hline Total & 78 & 66 & 108 & 24 & & 183 & 93 & & 276 \\
\hline
\end{tabular}

$R A$, right atrial; $R A D$, radiology; $I M$, internal medicine, AP, attending physicians.

${ }^{*} p \leq 0.05$ indicates significance.

an intranet-based survey tool for distribution. This software allows the survey to be delivered and completed online, with response data collated and exported in an electronic format that is compatible with SPSS 24 (IBM, SPSS Inc., Chicago, IL, USA). The survey link was sent to ICU physicians, radiologists, nephrologists, anesthesiologists, and senior medical residents. Study participation was optional, and responses were anonymized. There were no exclusion or disqualification criteria, and the data from all responders were included in the final analysis. The email addresses of all participants for questionnaire distribution were obtained from the departments for the five target medical disciplines: ICU, radiology, nephrology, anesthesia, cardiology and internal medicine. The survey link was simultaneously sent to all potential participants once, with two reminders to complete the survey that were sent out on alternate weeks. The data collection for this study was undertaken in April and May 2020.

\subsection{Outcomes of interest}

The outcomes of interest included a description of acceptable CVC tip positioning for all respondents and a description of acceptable positioning as a function of practice discipline and level of training. The between-group differences were evaluated by using a chi-square test, and all analyses were conducted in SPSS Statistics, with the level of significance defined by $\mathrm{p}<0.05$. 


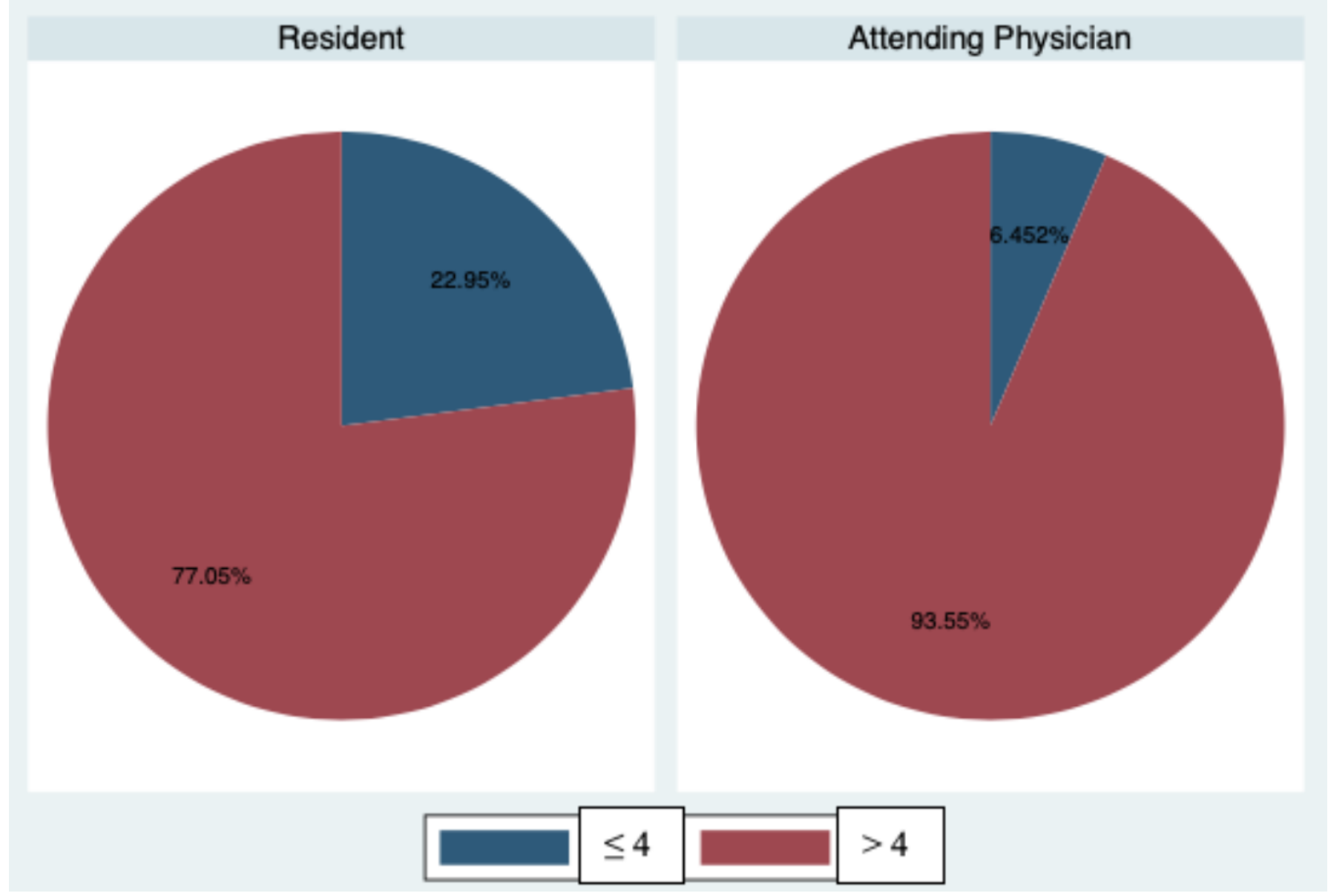

F I G U R E 4. Pie chart of a comparison of the preference of optimum central venous catheter tip position among residents and attending physicians. Attending physicians tended to have a wider range of acceptable central venous catheter tip range with regard to the carina $(>4 \mathrm{~cm})$ than did the residents $(p<0.001)$.

\section{Results}

During April and May 2020, a total of 276 physicians completed the survey (response rate 94.5\%). 78 ICU physicians, 66 radiologists, 108 internists, and 24 participants from "other" disciplines, including anesthesiologists, nephrologists, cardiologists, and emergency physicians were included. Among the 276 respondents, 183 were trainees with at least 3 months of ICU training. All respondents completed the survey within an average duration of $5 \mathrm{~min}$.

\subsection{Lower limit of CVC tip position}

The highest proportion 50\% (138/276) of physicians, regardless of their discipline and the training level, considered a threshold of $+4 \mathrm{~cm}$ ( $4 \mathrm{~cm}$ below the carina) as the lowest acceptable position for the CVC tip. This was the preferred position for $61.5 \%(48 / 78)$ of the ICU physicians, $59.1 \%$ $(39 / 66)$ of radiologists, and $38.9 \%(42 / 108)$ internists. However, $50 \%(12 / 24)$ of other specialties preferred the $+2 \mathrm{~cm}$ level ( $2 \mathrm{~cm}$ below the carina) as the lower acceptable position of the CVC tip. The preference for the CVC lower tip levels of 0 (internal medicine, $\mathrm{p}<0.001$ ), +2 (other specialties, $\mathrm{p}$ $=0.004$ ), and +4 (ICU and radiology, $\mathrm{p}=0.004$ ) differed significantly between departments (Table 1). Based on the level of training, a higher percentage of attending physicians
$61.3 \%$ (57/93) clearly preferred the +4 level ( $4 \mathrm{~cm}$ below the carina), compared with only $44.3 \%(81 / 183)$ of the residents ( $p$ $=0.007)$. At the level of the carina (0 level), senior residents preferred the 0 level compared to attending physicians ( $\mathrm{p}=$ 0.04; Table 1 and Fig. 2).

Besides the points referenced as the lower acceptable levels, we identified the cumulative numbers of individuals based on their preference of the lower CVC tip level - the zone between the chosen tip level and the carina - based on assumption that the individual choosing the lower acceptable level must be tolerant of the upper limit-level choices with regard to the carina. For example, if an individual accepts +4 then +2 and carina levels are acceptable. This range was referred as cumulative CVC lower limit. We observed a clear preference for $\leq+4$ among ICU and radiology participants $(\mathrm{p}<0.001)$ (Table 2). Based on the training level, the CVC cumulative level shows that attending physicians preferred a limit of $\leq$ +4 compared with the $\leq+2$ among the residents $(\mathrm{p}=0.04$ and 0.028 , respectively; Table 2 ).

The main reasons for catheter withdrawal, when found below the acceptable threshold, were the risk of arrhythmia $(50 \%$; $138 / 276$ ), followed by right atrial location regardless of the complications $(26.1 \% ; 73 / 276)$, risk of cardiac perforation $(16.3 \% ; 45 / 276)$, and risk of thrombosis $(7.6 \% ; 21 / 276)$. Furthermore, $30.8 \%$ of ICU physicians $(24 / 78)$ considered risk of 
TA B L E 4. Acceptable upper limit of CVC tip position based on the speciality and the level of experience (individual CVC tip-carina distance).

\begin{tabular}{lccccccccc} 
UA - CVC & ICU n (\%) & RAD n (\%) & IM n (\%) & Others n (\%) & p - value & Resident n (\%) & AP n (\%) & p - value & Total n (\%) \\
\hline$-\mathbf{2}$ & $18(23.1)$ & $18(27.3)$ & $54(50.0)$ & $3(12.5)$ & $<0.001^{*}$ & $81(44.2)$ & $12(12.9)$ & $<0.001^{*}$ & $93(33.7)$ \\
$-\mathbf{4}$ & $33(42.3)$ & $30(45.5)$ & $42(38.9)$ & $15(65.5)$ & 0.203 & $72(39.3)$ & $48(51.6)$ & 0.052 & $120(43.5)$ \\
\hline-6 & $27(34.6)$ & $18(27.3)$ & $12(11.1)$ & $6(25.0)$ & $0.002^{*}$ & $30(16.4)$ & $33(35.5)$ & $<0.001^{*}$ & $63(23.8)$ \\
\hline Total & 78 & 66 & 108 & 24 & & 183 & 93 & & 276
\end{tabular}

$U A$ - CVC, upper acceptable central venous catheter tip limit; $R A D$, radiology; IM, Internal medicine; AP, attending physicians; ICU, intensive care unit. ${ }^{*} p \leq 0.05$ indicates significance.

TA B L E 5. Acceptable upper limit of CVC tip position based on the speciality and the level of experience (cumulative CVC tip-carina distance).

\begin{tabular}{|c|c|c|c|c|c|c|c|c|c|}
\hline $\begin{array}{l}\text { CUA - CVC, } \\
\leq \text { level }\end{array}$ & ICU n (\%) & RAD & IM & Othe & $\mathbf{p}-\mathbf{r}$ & Resident & AP n (\%) & p - value & Tot: \\
\hline$\leq-2$ & $78(100)$ & $66(100)$ & $108(100)$ & $24(100)$ & N/A & $183(100)$ & $93(100)$ & N/A & $276(100)$ \\
\hline$\leq-4$ & 60 (76.9) & $48(72.8)$ & $54(50.0)$ & $21(80.5)$ & $<0.001^{*}$ & $102(55.7)$ & $81(87.1)$ & $<0.001^{*}$ & $183(66.3)$ \\
\hline$\leq-6$ & $27(34.6)$ & $18(27.3)$ & $12(11.1)$ & $6(25.0)$ & $0.002^{*}$ & $30(16.4)$ & $33(35.5)$ & $<0.001^{*}$ & $63(23.8)$ \\
\hline
\end{tabular}

$C U A$ - CVC, cumulative upper acceptable central venous catheter tip limit; RAD, radiology; IM, Internal medicine; AP, attending physicians; ICU, intensive care unit. ${ }^{*} p \leq 0.05$ indicates significance.

TA B L E 6. Preferred action if the CVC tip is above the acceptable limit.

\begin{tabular}{|c|c|c|c|c|c|c|c|c|c|}
\hline & ICU n (\%) & RAD n (\%) & IM n (\%) & Others n (\%) & p-value & Resident n (\%) & AP n (\%) & p-value & Total n (\%) \\
\hline New CVC ${ }^{a}$ & $27(34.6)$ & $3(4.6)$ & $33(30.6)$ & $9(37.5)$ & $<0.001^{*}$ & $54(29.5)$ & $18(19.4)$ & 0.069 & $72(26.1)$ \\
\hline No action & $9(11.5)$ & $18(27.3)$ & $6(5.6)$ & $6(25.0)$ & $<0.001^{*}$ & $21(11.5)$ & $18(19.4)$ & 0.076 & $39(14.1)$ \\
\hline Replace CVC ${ }^{b}$ & $42(53.9)$ & $45(68.2)$ & $69(63.9)$ & $9(37.5)$ & $0.032^{*}$ & $108(59.0)$ & $57(61.2)$ & 0.716 & $165(59.8)$ \\
\hline Total & 78 & 66 & 108 & 24 & & 183 & 93 & & 276 \\
\hline
\end{tabular}

${ }^{a}$ New catheter insertion, ${ }^{b}$ Replacement of the catheter over a guidewire within 24 hours. $R A D$, radiology; IM, Internal medicine; AP, attending physicians; ICU, intensive care unit.

$*_{p} \leq 0.05$ indicates significance.

cardiac perforation to be the main reason for catheter withdrawal compared with the other specialties $(p<0.001)$. Participants from internal medicine and radiologists considered risk of arrhythmia to be the most important reason for catheter withdrawal $(63.9 \%, 69 / 108$ and $59.1 \%, 39 / 66$, respectively; $\mathrm{p}<0.001)$. From the perspective of the level of training, the commonest reason for catheter withdrawal was the risk of arrhythmia among residents $(59 \% ; 108 / 183$; $\mathrm{P}<0.001)$. Attending physicians tend to be more concerned of the risk of cardiac perforation compared to residents $(32.3 \% ; 30 / 93$ and $8.2 \% ; 15 / 183$, respectively) $(\mathrm{p}<0.001)$ (Table 3$)$.

In case of catheter tip position below the acceptable level, most of the physicians $(46.7 \%$; 129/276) considered it moderately important to act when the CVC tip was below the optimal level. Most of the internal medicine respondents $(61.1 \%$; 66/108) were more concerned about the CVC tip below their optimal position than respondents from the ICU, radiology and other specialties. Among residents, the majority (55.7\%; $102 / 183$ ) considered it moderately important to withdraw the catheter $(\mathrm{p}<0.001)$, whereas among attending physicians, the majority $48.4 \%$ (45/93) considered it slightly important to withdraw the catheter $(p=0.002)$ in case of non-optimal tip position.

\subsection{Upper limit of the CVC tip position}

Most of the respondents $(43.5 \% ; 120 / 276)$ accepted $-4(4 \mathrm{~cm}$ above the carina) to be the upper limit, followed by $-2(33.7 \%$; 93/276). Furthermore, 50\% (54/108) of the internal medicine physicians chose -2 to be the upper acceptable limit for the CVC tip compared with respondents from the ICU, radiology, and other specialties $(\mathrm{p}<0.001)$. In contrast, intensivists tended to accept -6 as an upper acceptable CVC tip limit compared with respondents from radiology, internal medicine, and other specialties ( $p=0.002$; Table 4 ). Based on the level of training, majority of the residents $(44.2 \% ; 81 / 183)$ selected -2 as the upper limit $(\mathrm{p}<0.001)$, whereas majority of the attending physicians $(35.5 \%$; 33/93) chose -6 as the upper limit compared with only $16.4 \%(30 / 183)$ of the residents ( $p$ $<0.001$; Table 4 and Fig. 3).

Similar to the cumulative CVC lower limit, the cumulative CVC upper limit was also identified. The majority of physicians accept the $\leq-4$ level. Most of the physicians [76.9\% (60/73) ICU, 72.8\% (48/66) radiology, and 80.5\% (21 of 24$)$ others] tended to accept the $\leq-4$ level compared with $50 \%(54 / 108)$ of the internal medicine physicians $(\mathrm{p}<0.001)$. However, the attending physicians tended to accept $\leq-4$ (P $<0.001)$ and $\leq-6(\mathrm{p}<0.001)$ levels more than those among 
TA B L E 7. Optimal range of the CVC tip position around the landmark based on clinical subdiscipline and training level.

\begin{tabular}{|lcccccccccc}
\hline Optimal tip range & ICU n (\%) & RAD n (\%) & IM n (\%) & Others n (\%) & p-value & Resident n (\%) & AP n (\%) & p-value & Total n (\%) \\
\hline$\leq 4$ & $6(7.7)$ & $3(4.6)$ & $39(36.1)$ & 0 & $<0.001^{*}$ & $42(23.0)$ & $6(6.5)$ & $0.001^{*}$ & $198(71.7)$ \\
\hline$>4$ & $72(92.3)$ & $63(95.5)$ & $69(63.9)$ & $24(100)$ & $<0.001^{*}$ & $141(77.0)$ & $87(93.5)$ & $0.001^{*}$ & $78(28.3)$ \\
\hline Total & 78 & 66 & 108 & 24 & & 183 & 93 & 276 \\
\hline
\end{tabular}

$R A D$, radiology; IM, internal medicine; AP, attending physicians; ICU, intensive care unit.

$*_{p} \leq 0.05$ indicates significance.

the residents (Table 5).

Most of the physicians $(59.8 \% ; 165$ of 276) chose to replace the catheter of a new CVC, with $26.1 \%$ (72/276) choosing to insert a new catheter; however, $14.1 \%(39 / 276)$ preferred no action as long as all ports are working. Nonetheless, the respondents from all clinical specialties (ICU, internal medicine, and others) considered new catheter insertion, compared with radiologists who recommend no action $(\mathrm{p}<0.001)$. Among the training levels, the difference among residents and attending physicians with regard to a preference for catheter replacement was not significant for inserting new catheter $(p$ $=0.069)$, replacing new catheter $(\mathrm{p}=0.716)$, or no action $(\mathrm{p}=$ 0.076) (Table 6).

\subsection{Reference landmarks used and range for optimal CVC tip position}

The width of optimal range around was identified from the individual responses to assess their flexibility in accepting the range of the CVC tip position. The majority $(82.6 \% ; 228 / 276)$ of specialties have their optimal CVC tip range of $>4 \mathrm{~cm}$; the percentage of respondents with this preference among all specialties was more than $90 \%$, except in internal medicine with $63.6 \%(\mathrm{p}<0.001)$. However, the optimal CVC tip position range was narrow $(\leq 4 \mathrm{~cm})$ in $36.1 \%(39 / 108)$ of internists than in the other specialties ( $p<0.001$; Table 7 ). Based on the level of training, residents tend to prefer the CVC tip position in a narrow range as opposed to attending physicians, who preferred a wider range ( $p<0.001$; Fig. 4$)$.

The carina was the most commonly used landmark to determine the optimal CVC tip position in $71.7 \%(198 / 276)$ of respondents. However, $50 \%$ of radiologists $(33 / 66)$ used "other" landmarks, including the SVC and RA (40\%; 30/66) and the right mainstem bronchus $(10 \% ; 6 / 66)$.

\section{Discussion}

To the best of our knowledge, this is the first study to describe the knowledge, attitudes, and practice around optimal positioning of the CVC tip by using an objective assessment on a sample chest radiograph. In addition, this study showed a tendency of physicians from all specialties and levels of experience for making decisions based on their prior knowledge or previous experience, especially with regard to the complications of the CVC tip location based on a reading of the chest radiograph. In their review of 25 cases of CVCrelated pericardial tamponade, Collier et al. [8] identified that a position of the CVC tip within the cardiac silhouette was the commonest cause of tamponade. However, these previous studies did not report upon the position of the CVC tip relative to the carina or any other known and frequently used landmarks in the post-procedural chest radiographs. Moreover, $92 \%$ of CVCs used in those cases were stiff, with a catheter length approaching $30 \mathrm{~cm}$, compared with a shorter (typically $16 \mathrm{~cm}$ ) and softer catheter that is presently used. Most of the previously published studies [9-12] focused on simple calculations and various methods that used anthropometric measures to ensure optimal CVC tip placement. In a prospective study, Peres et al. [9] used anthropometric measurements and sought to determine the relationship between body height $(\mathrm{cm})$ and the ideal catheter length $(\mathrm{cm})$ for an appropriate CVC tip position. These catheter length formulas were subsequently validated by Czepizak et al. [10]. Those authors reported an efficiency of the equations to define the optimal and acceptable catheter length of $95 \%$, with the highest accuracy for estimating the catheter length observed with regard to the cannulation of the subclavian vein. These equations, however, and especially after introducing the new catheter length, have been debated in a recent study, with the sensitivity of Peres' original formulas, and derivatives of these as being $44 \%$ and $61 \%$, respectively [11].

Several methods have been proposed to improve the predictive accuracy of the length of the required catheter for optimal tip placement above the origin of the RA. A study compared outcomes for the placement of a predetermined 15$\mathrm{cm}$ catheter with that of a catheter with a customized length that was estimated on the basis of the distance between the thyroid notch to the sternal manubrium [12]. CVC repositioning was required in $2 \%$ of cases wherein a customized catheter length was used, compared with $78 \%$ in the predetermined $(15 \mathrm{~cm})$ catheter length group.

Gebhard et al. [13] reported a 96\% success rate of the correctly positioned CVC under electrocardiographic (ECG) guidance, compared with $75 \%$ in the non-ECG group, with correct positioning defined as the placement of the CVC tip in the middle of the SVC. Moreover, catheter repositioning was required in three patients in the ECG guidance group, compared with 20 patients in the non-ECG group. Similarly, McGee et al [14] reported a higher rate of successful placement of a 20-cm CVC by using a right atrial ECG technique compared with non-ECG guidance (conventional method). Notably, the accuracy rate with ECG guidance was comparable to that for the landmark method used for calculating the catheter length, based on the distance from the puncture site to the sternal notch and the level of the carina [15]. 
Clinical judgment alone is a poor predictor of tip position, with a sensitivity and specificity of $71 \%$ and $44 \%$, respectively. Furthermore, current practice guidelines do not recommend CVC use based on clinical judgment even if predetermined anthropometric calculations are used, because of the variability of the individual anatomical measurements as well as the change of patient postures, especially with frequent nursing care [16]. However, the timing of post-insertion chest radiography and the frequency of follow-up radiographs to detect possible migration have not been evaluated in clinical trials. The current standard of care is to perform chest radiography quickly after catheter placement and to look for possible migration if it is clinically indicated. A study evaluating the role of contrast-enhanced ultrasonography to determine the catheter tip position in the ICU reported the non-inferiority of this technique relative to chest radiography, with a sensitivity and specificity of $96 \%$ and $93 \%$, respectively [17]. However, more studies are needed to thoroughly assess the effectiveness of venous ultrasonography to determine the catheter tip location.

Most guidelines strongly advise against the placement of the CVC tip in the distal half of the SVC or SVC-RA, and the confirmation of tip placement through bedside chest radiography has been associated with high interobserver variability [18]. Historically, several skeletal radiologic and surface landmarks, SVC, SVC-RA, and RA have been proposed to identify the appropriate CVC tip position, although all were found to be misleading because of the "parallax" effect which is the variable effect of x-ray beams on the position and size of the skeletal structures [19-21]. Aslamy et al. and Schuster et al. proved the usefulness of the carina as the most reliable landmark by using magnetic resonance imaging and cadaver examination, respectively $[22,23]$. This study revealed that nearly $71 \%$ of the respondents used the carina as the only landmark while the rest of them used it in conjunction with other landmarks.

This study demonstrated that the decision to withdraw the CVC tip if it was below the acceptable level, as opposed to leaving it in place, was less necessary among more than half of ICU attending physicians, with physicians from other disciplines indicating that catheter withdrawal would be important if the tip was in this low position. Most ICU physicians reported that catheter withdrawal was unimportant if the patient was asymptomatic, as the catheters that are presently in use are soft, which significantly lowers the risk of complications.

Several complications have been reported to result from malpositioning of the CVC tip or migration of the tip from the initial placement position. Despite its infrequent occurrence, cardiac tamponade remains the most serious of the complications associated with the malpositioning of the CVC tip [24]. In this study, we found that most intensivists realized the risk of cardiac perforation and tamponade with the malpositioning of the CVC tip. Factors that may play a role in the development of cardiac tamponade include penetration of the pericardial sac by the dilator and guidewire, catheter stiffness, angle of the catheter tip to the vessel wall, especially for left-sided catheters, and infusion of hyperosmolar solutions [24, 25]. Over the last two decades, with the use of newer catheters, only nine cases of localized cardiac tamponade have been reported, and all of these were associated with the insertion of Swan-
Ganz catheters, dialysis lines, and Port-A-Caths [26-32]. Of note, the tip position was not reported in these case reports.

Thrombosis either around the catheter tip or in the proximal part of the catheter is an ICU complication that may result from catheter malposition, and can normally be detected as early as the third day after CVC placement [33].Multiple studies have reported a catheter-related thrombosis if the catheter tip was located in the proximal SVC or distally situated in the RA, compared with that with a CVC tip within the distal SVC and the SVC-RA junction [34-36].

In this study, the decision of approximately half of the physicians to withdraw the catheter if the tip was placed below the lower acceptable level was based on the risk of arrhythmias. Transient arrhythmias are likely to occur from the insertion of the guidewire. Most of the reported arrhythmias were atrial and ventricular premature beats and bigeminy [37]. However, supraventricular and ventricular tachycardia is more likely to occur in patients with reduced cardiac function [38, 39].

The futility of the post-procedural chest radiograph for dialysis catheter tip confirmation has been addressed in one review due to the risk of unnecessary radiation exposure compared to the lower risk of complications from the CVC cannulation and the malposition of CVC tip after the procedure. However, the necessity of chest radiographs in the critical care areas outweighs the harm from the radiation exposure because of other indications such as the frequent use of invasive and non-invasive mechanical ventilation [40]. The positive intrathoracic pressure with the variability of pressure and volume settings theoretically may increase the chances on malposition and may also lead to more complications associated with CVC insertion; hence the importance of follow up chest radiograph. Nevertheless, there is also a medicolegal implication by which the CVC tip confirmation is important if the chest radiographs were to be done due to other indications.

\subsection{Limitations}

The limitations of our study need to be acknowledged in the interpretation of the findings. The majority of the participants were senior medical residents, most of whom had a practical approach toward managing the catheter tip position. Nonetheless, it was necessary to include trainees in this survey to ascertain their clinical judgment. With regard to the senior physicians, only a few nephrologists participated in this study, and the inclusion of a higher number of nephrologists would have facilitated an understanding of their clinical attitude toward the malposition of the short-term non-tunneled dialysis catheter, and to comparatively evaluate possible differences in their practice patterns with those of the intensivists. Moreover, we did not ascertain the factors associated with physician awareness of tip malposition, such as the selection of the insertion site, catheter length, and the route of insertion.

Future research is needed to identify the acceptable range of the catheter tip position based on real-time or fixed-length measurements from familiar landmarks on the chest radiograph instead of subjective assessment as well as to readdress the number and types of complications encountered during the care of the CVC tip, especially with regard to the improvement of CVC composition and quality and advances in the man- 
ufacturing technology and design of CVC kits over the last decade. Large-sample, high-quality studies will facilitate improvements to develop a more practical and efficient approach for application in clinical practice.

\section{Conclusion}

The optimal position and adjustment of the CVC tip position remain an overlooked topic in the ICU and in all critical care areas. Nonetheless, this study concludes that the majority of physicians in this study accepted the lower and upper limits for the CVC tip to be $4 \mathrm{~cm}$ below and above the carina, respectively. A wider CVC tip range, however, tends to be more acceptable approach by the intensivists and more experienced physicians than physicians from other medical disciplines or less experienced physicians especially with the introduction of the new catheters. Additionally, the intensivists and more experienced physicians gave greater credence to the risk of cardiac perforation and tamponade.

\section{Highlights}

Central venous catheter tip positioning is influenced by physician preferences and landmarks.

Post-procedural chest radiography using digital tape measurement in the imaging software may facilitates early and late intervention of tip malpositioning.

Specific guidelines on the insertion and troubleshooting of central venous catheters especially for ICU patients are needed.

\section{Acknowledgements}

I would like to thank the participating departments at the King Saud University-affiliated teaching hospitals for their valuable help and support. I would like to thank all the peer reviewers and editors for their opinions and suggestions.

\section{Conflict of interest}

The author has no conflicts of interest to declare.

\section{REFERENCES}

[1] Practice Guidelines for Central Venous Access 2020: An Updated Report by the American Society of Anesthesiologists Task Force on Central Venous Access. Anesthesiology. 2020;132:8-43.

[2] Abrams HL. In: Baum S, Pentecost MJ, editors. Abrams' angiography. Interventional radiology, 2nd ed., Philadelphia: Lippincott Williams \& Wilkins; 2006, p.1142-1154.

[3] Pikwer A, Baath L, Davidson B et al. The incidence and risk of central venous catheter malpositioning: a prospective cohort study in 1619 patients. Anaesth Intensive Care. 2008;3:30-37.

[4] Lok CE, Huber TS, Lee T, et al. KDOQI Clinical Practice Guideline for Vascular Access: 2019 Update. Am J Kidney Dis. 2020;75:S1-S164.

[5] Bishop L, Dougherty L, Bodenham A et al. Guidelines on the insertion and management of central venous access devices in adults. Int $\mathrm{J}$ Lab Hematol. 2007;29:261-278.

[6] Pittiruti M, Hamilton H, Biffi R et al. ESPEN guidelines on parenteral nutrition: central venous catheters. Clin Nutr. 2009;28:365-377.

[7] Infusion Nurses Society. Infusion nursing standards of practice. J Infus Nurs. 2006;29:S1-92.

[8] Collier PE, Ryan JJ, Diamond DL. Cardiac tamponade from central venous catheters. Report of a case and review of the English literature. Am J Surg. 1998;176:212-214.

[9] Peres PW. Positioning central venous catheters: a prospective survey. Anaesth Intensive Care. 1990;18:536-539.

[10] Czepizak CA, O'Callaghan JM, Venus B. Evaluation of formulas for optimal positioning of central venous catheters. Chest. 1995;107:16621664.

[11] Kim WY. Optimal insertion depth of central venous catheters-is a formula required? A prospective cohort study. Injury. 2012;43:38-41.

[12] Ezri T, Weisenberg M, Sessler DI et al. Correct depth of insertion of right internal jugular central venous catheters based on external landmarks: avoiding the right atrium. J Cardiothorac Vasc Anesth. 2007;21:497-501.

[13] Gebhard RE, Szmuk P, Pivalizza EG et al. The accuracy of electrocardiogram controlled central line placement. Anesth Analg. 2007;104:65-70.

[14] McGee WT, Ackerman BL, Rouben LR et al. Accurate placement of central venous catheters: a prospective, randomized, multicenter trial. Crit Care Med. 1993;21:1118-1123.

[15] Lee JH, Bahk JH, Ryu HG et al. Comparison of the bedside central venous catheter placement techniques: landmark vs. electrocardiogram guidance. Br J Anaesth. 2009;102:662-666.

[16] Abood GJ, Davis KA, Esposito TJ et al. Comparison of routine chest radiograph versus clinician judgment to determine adequate central line placement in critically ill patients. J Trauma. 2007;63:50-56.

[17] Vezzani A, Brusasco C, Palermo S et al. Ultrasound localization of central vein catheter and detection of postprocedural pneumothorax: an alternative to chest radiography. Crit Care Med. 2010;38:533-538.

[18] Wirsing M, Schummer C, Neumann R et al. Is traditional reading of the bedside chest radiograph appropriate to detect intra-atrial central venous catheter position? Chest. 2008;134:527-533.

[19] Lee JB, Lee YM. Pre-measured length using landmarks on posteroanterior chest radiographs for placement of the tip of a central venous catheter in the superior vena cava. J Int Med Res. 2010;38:134-141.

[20] Defalque RJ, Campbell C. Cardiac tamponade from central venous catheters. Anesthesiology. 1979;50:249-252.

[21] Rutherford JS, Merry AF, Occleshaw CJ. Depth of central venous catheterization: an audit of practice in a cardiac surgical unit. Anaesth Intensive Care. 1994;22:267-271.

[22] Aslamy Z, Dewald CL, Heffner JE. MRI of central venous anatomy: implications for central venous catheter insertion. Chest. 1998;114:820 826.

[23] Schuster M, Nave H, Piepenbrock $\mathrm{S}$ et al. The carina as a radiological landmark in central venous catheter placement. $\mathrm{Br} \mathrm{J}$ Anaesth. 2000;85:192-194.

[24] Collier PE, Ryan JJ, Diamond DL. Cardiac tamponade from central venous catheters. Report of a case and review of the English literature. Angiology. 1998;35:595-600.

[25] Collier PE, Goodman GB. Cardiac tamponade caused by central venous catheter perforation of the heart: A preventable complication. J Am Coll Surg. 1995; 181:459-463.

[26] Antevil JL, Karavas AN, Byrne G et al. Pulmonary artery catheter deviation on chest roentgenogram after cardiac operation: a sign of tamponade. Ann Thorac Surg. 2010;89:281-283.

[27] Lee YM, Kim HJ, Lee JE et al. Cardiac tamponade following insertion of an internal jugular vein catheter for hemodialysis. Clin Nephrol. 2009;72:220-223.

[28] Ervo R, Angeletti S, Dertenois LT et al. Dialysis catheter associated cardiac tamponade: quick diagnosis by extemporaneous echocardiography. J Vasc Access. 2008;9:69-71.

[29] Schmiege LM, Wolf DA. A case of fatal cardiac tamponade during hemodialysis. Semin Dial. 2006;19:434-437.

[30] Quinn MP, Maxwell P, Brown JH et al. Cardiac tamponade ... a wire too far? Nephrol Dial Transplant. 2006;21:2669-2670.

[31] Smart PJ, Kirton M, Mann B. Cardiac tamponade during central venous vascular access port insertion. ANZ J Surg. 2005;75:617-618.

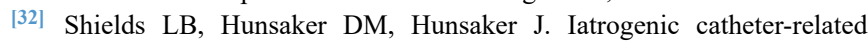
cardiac tamponade: a case report of fatal hydropericardium following subcutaneous implantation of a chemotherapeutic injection port. J Forensic Sci. 2003;48:414-418.

[33] Rash K, Rao SM, Badwaik G et al. Thrombosis associated with right 
internal jugular central venous catheters: A prospective observational study. Indian J Crit Care Med. 2012;16:17-21.

[34] Timsit JF, Farkas JC, Boyer JM et al. Central vein catheter-related thrombosis in intensive care patients. Chest. 1998;114:207-213.

[35] Puel V, Caudry M, Le Métayer P et al. Superior vena cava thrombosis related to catheter malposition in cancer chemotherapy given through implanted ports. Cancer. 1993;72:2248-2252.

[36] Gilon D, Schechter D, Rein AJ et al. Right atrial thrombi are related to indwelling central venous catheter position: insights into time course and possible mechanism of formation. Am Heart J. 1998;135:457-462.

[37] Stuart RK, Shikora SA, Akerman P et al. Incidence of arrhythmia with central venous catheter insertion and exchange. J Parenter Enteral Nutr. 1990;14:152-155.

[38] Yavascan O, Mir S, Tekguc H. Supraventricular tachycardia following insertion of a central venous catheter. Saudi J Kidney Dis Transpl.
2009;20:1061-1064

[39] Kasten GW, Owens E, Kennedy D. Ventricular tachycardia resulting from central venous catheter tip migration due to arm position changes: report of two cases. Anesthesiology. 1985;62:185-187.

[40] Parmar MS. (F)utility of "routine" postprocedural chest radiograph after hemodialysis catheter (central venous catheter) insertion. J Vasc Access. 2020;1129729820907259. doi:10.1177/1129729820907259.

How to cite this article: Rakan M. AlQahtani. A Novel Objective Approach Towards Physicians' Knowledge and Practice Patterns to Optimize Central Venous Catheter Placement: A Cross-sectional Study. Signa Vitae. 2020;16(2):124-133. doi:10.22514/sv.2020.16.0052. 